\title{
IDENTIFICATION OF MEGALOBLASTIC ANEMIA CELLS THROUGH THE USE OF IMAGE PROCESSING TECHNIQUES
}

\author{
Asaad Babker ${ }^{1}$, Vyacheslav Lyashenko². \\ ${ }^{1}$ Medical Laboratories Science Department, College of Health Science, Gulf Medical University, Ajman, UAE. \\ ${ }^{2}$ Department of Informatics, Kharkiv National University of RadioElectronics, Kharkiv, Ukraine.
}

\section{ABSTRACT}

Objective: Our aim is to show the possibility of using different image processing techniques for blood smear analysis. Also our aim is to determine the sequence of image processing techniques to identify megaloblastic anemia cells. Methods: We consider blood smear image. We use a variety of image processing techniques to identify megaloblastic anemia cells. Among these methods, we distinguish the modification of the color space and the use of wavelets. Results: We developed a sequence of image processing techniques for blood smear image analysis and megaloblastic anemia cells identification. As a characteristic feature for megaloblastic anemia cells identification, we consider neutrophil image structure. We also use the morphological methods of image analysis in order to reveal the nuclear lobes in neutrophil structure. Conclusion: We can identify the megaloblastic anemia cells. To do this, we use the following sequence of blood smear image processing: color image modification, change of the image contrast, use of wavelets and morphological analysis of the cell structure.

KEYWORDS: Image processing; Megaloblastic anemia cells; Wavelet analysis; Morphological analysis; Blood smear.

\section{INTRODUCTION}

Processing of microscope images in medicine is one of research areas priority. Among many medical imaging it is necessary to allocate the image of blood preparations. Blood is the source of information about the processes that occur in the human body. Blood consists of plasma, erythrocytes, leukocytes and platelets. Blood cellular structures changing may be an evidence of a person's disease. Therefore, the blood smear analysis is one of the methods for diagnosing human diseases, assessing the state of human health $[1,2]$.

Blood smear analysis is also important from the point of view of pharmaceutical science. We need to know how medications affect the cell and its structure. For this, we need to know the structure of the cell during the illness and the structure of the cell after using medications. We also need to know the exact structure of the cell in order to correctly diagnose the disease and correctly assign medications. It is important to know all the details of the cellular structure.

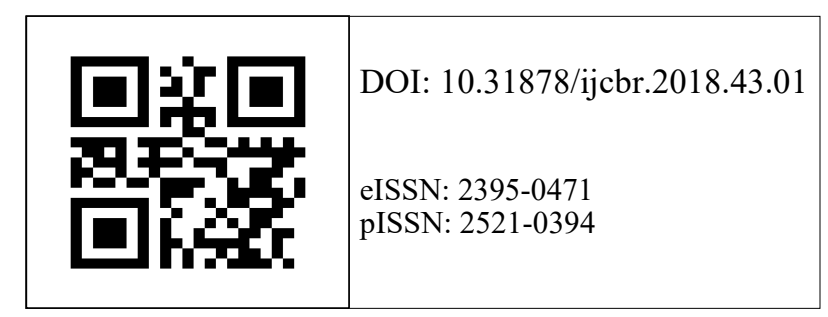

A typical example of a change in blood cellular structures is megaloblastic anemia. Megaloblastic anemia is a blood disorder marked by the appearance of very large red cells. As a consequence, erythrocytes do not function fully and therefore displace healthy cells [3]. This entails the appearance of a large number of immature and not fully developed other blood cells. It is possible to observe the complex structure of megaloblastic anemia cells [4]. The megaloblastic anemia cells are characterized by a large number of segments in the nucleus (hypersegmented neutrophil). In this normal neutrophils only contain three or four nuclear lobes (segments). Therefore it is important to know the internal structure of megaloblastic anemia cells.

One way to analyze a blood smear is to use image processing techniques [5-8]. Image processing is one of the areas of data mining and method for extracting additional information about processes under experimental study. These methods allow us to describe more accurately the change in the cellular structures of the blood. The uniqueness and individuality of each sample blood smear makes it necessary to use several stages of Image processing. This is necessary for qualitative analysis. A sequence of different imaging techniques is considered for blood smear analysis and identification of megaloblastic anemia cells.

Correspondence: Asaad Babker. Medical Laboratories Science Department, College of Health Science, Gulf Medical University, Ajman, UAE. Email: azad.88@hotmail.com 


\section{MATERIALS AND METHODS}

\section{Study design: An observation study}

Study location: Department of Informatics, Kharkiv National University of RadioElectronics, Kharkiv, Ukraine.

Study time frame: Local data.

Sample size: 5 blood films.

Inclusion criteria: Patient with megaloblastic anemia.

\section{Methodology:}

Various methods are used to analyze medical images. Among such methods it is necessary to allocate: contrast change, noise removal, object contour selection, segmentation, and objects identification. Each method is applied in strict accordance with the need for its use.

But these methods can be combined. Then we get a new result. The main methods for analyzing medical images are described below. This is the basis of our methodology. This is the basis for obtaining a new result.

The contrast change procedure is designed to improve the initial image quality. Changing the image contrast makes some details of the image clearer [79]. Therefore, changing the contrast is an important element in medical images analysis. This is especially important when we examine medical images that are obtained with the help of a microscope.

Noise removal is the removal of defects that are present in the image. Defects arise during medical image registration. But removing the defects, we can delete the details of the image. Therefore, this stage of medical images processing should be used very carefully.

Object contour selection is boundary selection for object that is being examined. For this purpose various methods are used to detect the contour $[7,8,10]$.

Segmentation is entire image separation into parts: objects and background [9]. This makes it possible to delete some information that is not important. Then we can speed up medical image processing.

Objects identification is objects detection on the image with the help of some properties: the size of the object, the brightness of the object, the shape of the object. Identification is also the recognition of an object by its properties. If we analyze megaloblastic anemia cells, identification is a study of the structure of megaloblastic anemia cells.

Among the methods of image processing we should also highlight: the transformation of color spaces, the ideology of image processing with the help of wavelets. These methods allow improving the quality of medical images processing and analysis. Wavelet analysis allows to highlight the characteristic features of medical images that are poorly visualized. This is due to the fact that wavelet processing allows taking into account the particular characteristics of the images under study by decomposing source data into a plurality of approximate and detail coefficients, in particular by image contour detection [7-9, 11]. Color spaces conversion allows improving the quality of the procedure for increasing medical image contrast. This is possible due to the fact that we analyze the individual components of a color image. Such an analysis improves the quality of the procedure for changing image contrast and the speed with which this procedure is performed.

\section{RESULTS}

For megaloblastic anemia cells identification, we will use the following sequence of blood smear image processing (Fig. 1, this sequence was suggested by the authors. It is developed on the basis of known methods):

1. We consider a color image as the original image. This allows us to examine visually the cellular structure in more details.

2. We are modifying the color space. Typically, a color medical image is represented in the RGB for-

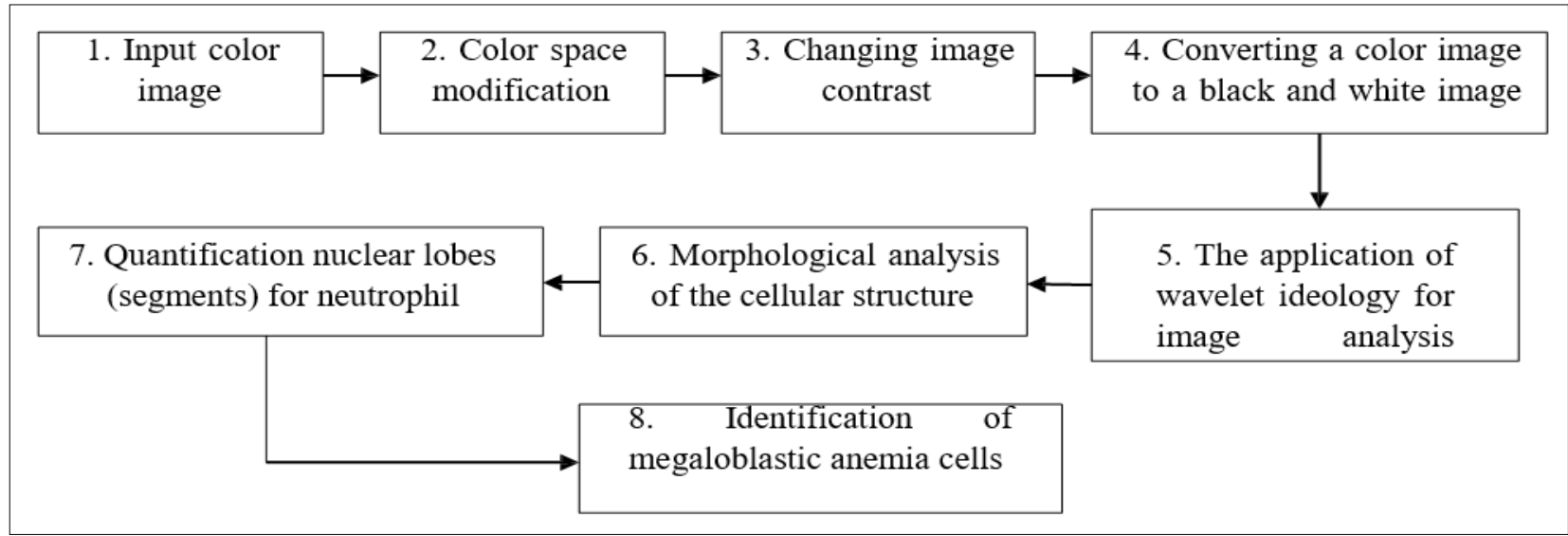

Figure 1. Sequence of Blood Smear Image Processing Steps for Identification of Megaloblastic Anemia Cells 


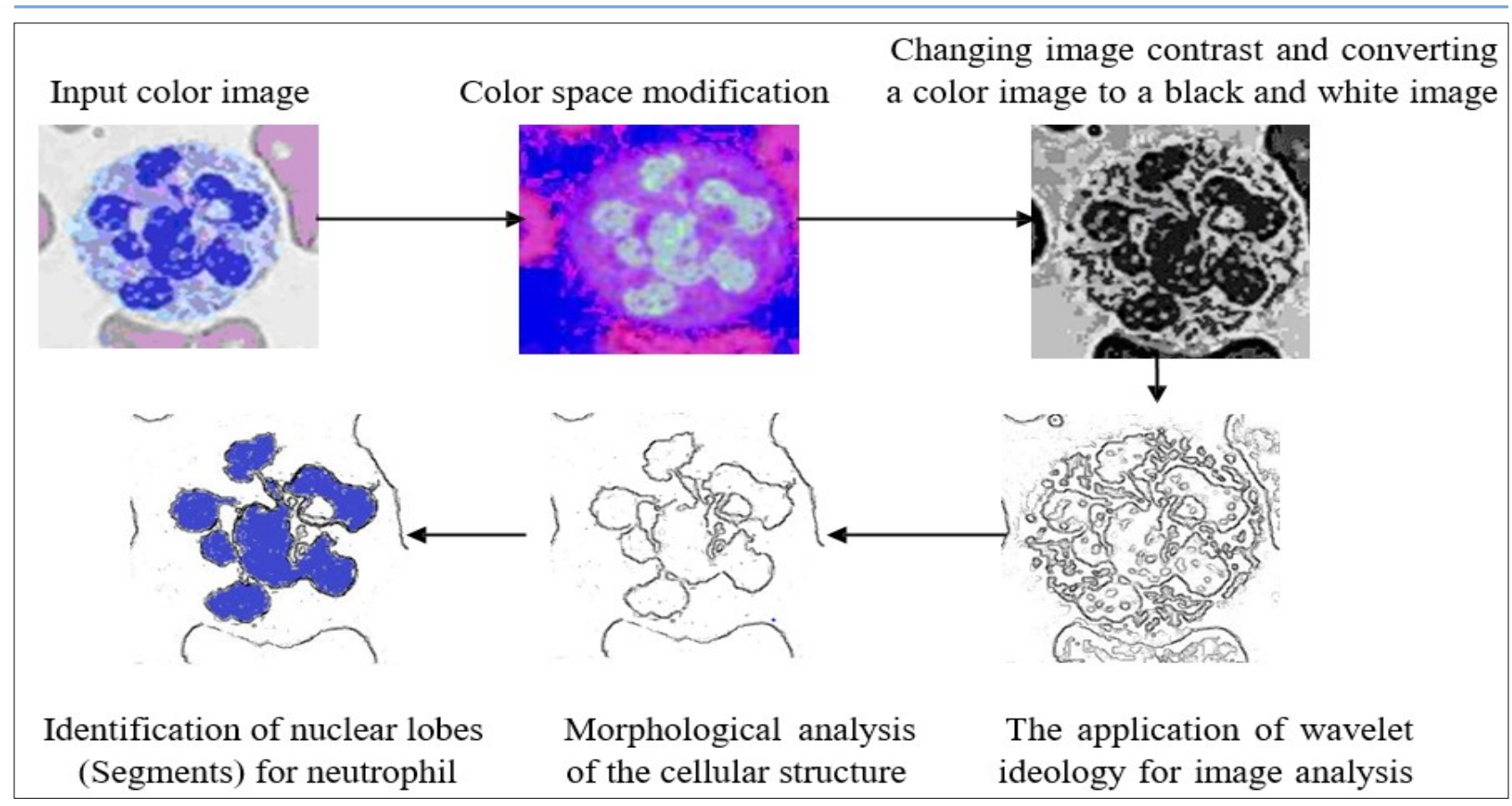

Figure 2. Some Results of Blood Smear Processing for Identification of Megaloblastic Anemia Cells

mat: R (red), G (green) and B (blue). In this way most systems for medical images recording and analyzing work [9]. But for analysis we can use other systems of color image, for example, it can be HSV color system: channels-H (hue), S (saturation), V (volume or brightness) [12]. Such a transition allows us to emphasize the features of the cellular structure because of the difference in perception and representation of the image points for different color spaces. This is due to the fact that the HSV system is a nonlinear conversion of the RGB system. Thus, we distinguish the hidden features of the cellular structure.

3. Change in contrast. We apply the method of histogram change in the specified range with gamma correction. We conducted studies that showed that the histogram change in the specified range with gamma correction combined with the conversion of color space from RGB to HSV increases the contrast of the original image by an order in comparison with other methods [12]. This result is associated with emphasizing the low contrast areas of the original image due to non-linear transformation of the original image (color space modification) and subsequent non-linear correction of the converted image.

4. We move from a color image to a black and white image. Such a transition means that each point of the original (color) image has not three RGB or HSV coordinates, but one coordinate that varies in luminance levels from 0 to $256[9,11]$. This is the standard procedure for moving from a color image to a black and white image. This procedure is necessary for subsequent actions - the application of wavelet analysis.
5. Wavelet analysis. We use wavelet analysis to detect the contour of cell structure elements. We consider the image as a data set, to which we apply the wavelet function (the debouchy wavelet-2). This wavelet function allows us to select the outline of all original image elements. The wavelet analysis procedure is described in detail by Lyashenko et al. [1, 8, 12], Kobylin and Lyashenko [11].

6. Morphological analysis of the cellular structure includes: the removal of single contour lines, the removal of small objects and the union of compact contour lines. For this, methods of morphological analysis of contour image elements are used. We use standard procedures:

Combining points in a line if the points of the contour are at a given distance from each other delete points if the counter points are far apart. This helps to identify the nuclear lobes in the structure of the neutrophil, and also reveal the number of nuclear lobes.

7. Determination of the number of nuclear lobes (segments) for neutrophil. For this, we combine the original image with the image after the morphological analysis. So we get only the image of the nuclear lobes (segments). This allows us to determine the number of nuclear lobes (segments) in the image.

8. If we know the number of nuclear lobes (segments) for neutrophil, we can identify Megaloblastic anemia Cells. This is important for determining the disease and choosing medications.

Fig. 2 presents some results of blood smear processing for identification of megaloblastic anemia cells. Each result in Fig. 2 corresponds to a certain stage in Fig.1. 
The ideology of stages corresponds to the basic methods of image analysis.

\section{DISCUSSION}

Image processing is one of the tools for the analysis of cellular structures. Therefore, there are many different studies where the ideology of image processing for processing medical images is used.

For example, M. Saha et. al. [13] suggest using the threshold segmentation to isolate the cell nucleus. N. Dey et al. [9] and G. Mahendran et al. [14] also discuss the segmentation issues of cells cytology preparations images using a threshold. But for this you need to know the threshold level. If we process image, then the threshold level will also change. Therefore, to improve image quality, we suggest using the method histogram change in the specified range with gamma correction [12]. This allows us to get a good level of contrast for the image and make the details of our image clearer. This is important for image segmentation. A lot of research is devoted to the problem of choosing a threshold $[9,15,16]$. But as practice shows, the problem of choosing a threshold arises again. Therefore, it is better to change the contrast of the image and then carry out segmentation of the image. This makes it possible not to make the procedures for images processing that do not provide optimal results. Thus, we increase the speed of image processing and reduce the time for obtaining the result.

S. Singh and R. Gupta [17] suggest using filtering to improve image quality. But then we can also delete the details of the image. Therefore, we offer only the use of image contrast change. In this case, we will improve the quality of the original image and save all the details of the image. This will increase the reliability of the analysis with the help of image processing ideology. Our estimates show: we do not lose the details of the original image (even very small ones); we improve the quality of segmentation by $20 \%$ in comparison with the classical approaches.

For segmentation we use the ideology of wavelet analysis [12]. This avoids the errors that arise in the case of classical segmentation. We can also select very small objects in the source image. This allows us to improve the accuracy of the procedure for original image processing and improve the accuracy of the results.

We also use morphological analysis to improve the quality of the result. This analysis is the final stage of our image processing procedure. At the same time Malviya et al. [16] use morphological analysis at the initial stage of segmentation. But this leads to segmentation errors, because that there may be some ambiguity while localizing nucleus [16].
An important feature of image processing for blood smear analysis is the emerging difference in the relative staining intensity of the clinical samples examined. This is said by many authors who use the ideology of image processing for the analysis of medical images $[9,16,18]$. Therefore, in our procedure color image modification stage is used [12]. This allows us to take into account the peculiarities of staining of the studied clinical samples.

In general, we have received a new procedure for medical images processing, which is based on image processing ideology. Medical images processing procedure allows improving the quality and reliability of the results of initial images analysis.

\section{CONCLUSIONS}

Obtained results can be used to identify megaloblastic anemia cells automatically. This is important for determining the disease and choosing medications. We showed that for this it is necessary to use a certain sequence of image processing methods. We justified the selection of a certain sequence of medical image processing for the identification of megaloblastic anemia cells. Different stages of the image processing procedure were considered and the best steps for image analysis of megaloblastic anemia cells were selected. In this case, important are: Modification of color space. This allows a qualitative increase in the contrast of the blood smear image; Use of wavelets. This allows a qualitative analysis of the structure of neutrophil image. We examined a method that improves the accuracy of diagnosis of the disease and improves the quality of medications selection.

\section{Conflict of interest: Nil}

\section{REFERENCES}

1. Li H, Colin S. Characteristic peripheral blood smear findings in disorders of cobalamin metabolism. Blood 2016;128(21): 2584-2584.

2. Chari PS, Prasad S. Pilot Study on the Performance of a New System for Image Based Analysis of Peripheral Blood Smears on Normal Samples. Indian Journal of Hematology and Blood Transfusion. 2017: 1-7.

3. Yamamoto A, Kambara $\mathrm{Y}$, Urata $\mathrm{T}$, Kuroi $\mathrm{T}$, Masunari T, Sezaki N, Kiguchi T. Mean Corpuscular Volume Evaluation for Differential Diagnosis of Myelodysplastic Syndrome and Megaloblastic Anemia: A Study of 130 Patients. Clinical Lymphoma, Myeloma and Leukemia. 2017;17: S346S347.

4. Bizzaro N, Antico A. Diagnosis and classification of pernicious anemia. Autoimmunity reviews. 2014; 13(4): 565-568. 
5. Putzu L, Caocci G, Di Ruberto C. Leucocyte classification for leukaemia detection using image processing techniques. Artificial intelligence in medicine. 2014; 62(3): 179-191.

6. Tomari R, Zakaria WNW, Jamil MMA, Nor FM, Fuad NFN. Computer aided system for red blood cell classification in blood smear image. Procedia Computer Science. 2014;42:206-213.

7. Lyashenko V, Babker AMAA, Kobylin OA. The methodology of wavelet analysis as a tool for cytology preparations image processing. Cukurova Med J. 2016; 41(3): 453-463.

8. Lyashenko V, Matarneh R, Kobylin O, Putyatin Y. Contour Detection and Allocation for Cytological Images Using Wavelet Analysis Methodology. International Journal of Advance Research in Computer Science and Management Studies. 2016;4: 85-94.

9. Dey N, Ashour AS, Ashour AS, Singh A. Digital analysis of microscopic images in medicine. Journal of Advanced Microscopy Research. 2015; 10: 1-13.

10. Xiong W, Ong SH, Lim JH, Cheng J, Gu Y Blood Smear Analysis, Malaria Infection Detection, And Grading From Blood Cell Images. Biomedical Image Understanding, Methods and Applications 2015:275-324.

11. Kobylin O, Lyashenko V. Comparison of standard image edge detection techniques and of method based on wavelet transform. International Journal of Advanced Research.2014; 2(8): 572-580.

12. Lyashenko V, Babker AM. Using of Color Model and Contrast Variation in Wavelet Ideology for Study Megaloblastic Anemia Cells. Open Journal of Blood Diseases. 2017; 7(03): 86-102.

13. Saha M, Agarwal S, Arun I, Ahmed R, Chatterjee S, Mitra P, Chakraborty C. Histogram Based Thresholding for Automated Nucleus Segmentation Using Breast Imprint Cytology. Advancements of Medical Electronics. 2015: 49-57.

14. Mahendran G, Babu R, Sivakumar D. Automatic segmentation and classification of pap smear cells. International Journal of Management, IT and Engineering. 2014; 4(5): 100-108.

15. Ensink E, Sinha J, Sinha A, Tang $H$, Calderone HM, Hostetter G, Haab BB. Segment and Fit Thresholding: A New Method for Image Analysis Applied to Microarray and Immunofluorescence Data. Analytical chemistry 2015;87(19): 9715-9721.
16. Malviya R, Karri SPK, Chatterjee J, Manjunatha M, Ray AK. Computer assisted cervical cytological nucleus localization. TENCON 2012-2012 IEEE Region 10 Conference. IEEE 2012: 1-5.

17. Singh S, Gupta R. Identification of components of fibroadenoma in cytology preparations using texture analysis: a morphometric study. Cytopathology 2012; 23(3): 187-191.

18. Al-Kofahi Y, Lassoued W, Lee W, Roysam B. Improved automatic detection and segmentation of cell nuclei in histopathology images. Biomedical Engineering. IEEE Transactions on 2010; 57 (4): 841-852.

How to Cite this article: Asaad Babker, Vyacheslav Lyashenko. Identification of megaloblastic anemia cells through the use of image processing techniques. Int. j. clin. biomed. res. 2018;4(3): 1-5. 